\title{
Targeting adhesion signaling in KRAS, LKB1 mutant lung adenocarcinoma
}

\author{
Melissa Gilbert-Ross, ${ }^{1,2}$ Jessica Konen, ${ }^{1,2}$ Junghui Koo, ${ }^{1,2}$ John Shupe, ${ }^{1,2}$ Brian S. Robinson, ${ }^{3}$ \\ Walter Guy Wiles IV, ${ }^{2,4}$ Chunzi Huang, ${ }^{2,4}$ W. David Martin, ${ }^{1}$ Madhusmita Behera, ${ }^{5}$ \\ Geoffrey H. Smith, ${ }^{3}$ Charles E. Hill, ${ }^{3}$ Michael R. Rossi, ${ }^{3,6}$ Gabriel L. Sica, ${ }^{3}$ Manali Rupji, ${ }^{2}$ \\ Zhengjia Chen, ${ }^{2,7}$ Jeanne Kowalski, ${ }^{2,7}$ Andrea L. Kasinski, ${ }^{8}$ Suresh S. Ramalingam, ${ }^{1,2}$ Haian Fu, ${ }^{9,2}$ \\ Fadlo R. Khuri, ${ }^{1,2}$ Wei Zhou, ${ }^{1,2,3,10}$ and Adam I. Marcus ${ }^{1,2}$ \\ 'Department of Hematology and Medical Oncology, Emory University School of Medicine, ${ }^{2}$ Winship Cancer Institute of \\ Emory University, ${ }^{3}$ Department of Pathology and Laboratory Medicine, Emory University School of Medicine, \\ ${ }^{4}$ The Cancer Animal Models Shared Resource, ${ }^{5}$ Winship Research Informatics Shared Resource, Winship Cancer \\ Institute, ${ }^{6}$ Department of Radiation Oncology, Emory University School of Medicine, 'Department of Biostatistics and \\ Bioinformatics, Rollins School of Public Health, Emory University, Atlanta, Georgia, USA. ${ }^{8}$ Department of Biological \\ Sciences, Purdue University, West Lafayette, Indiana, USA. ${ }^{9}$ Department of Pharmacology, Emory University School of \\ Medicine, ${ }^{10}$ Department of Human Genetics, Emory University, Atlanta, Georgia, USA.
}

Loss of LKB1 activity is prevalent in KRAS mutant lung adenocarcinoma and promotes aggressive and treatment-resistant tumors. Previous studies have shown that LKB1 is a negative regulator of the focal adhesion kinase (FAK), but in vivo studies testing the efficacy of FAK inhibition in LKB1 mutant cancers are lacking. Here, we took a pharmacologic approach to show that FAK inhibition is an effective early-treatment strategy for this high-risk molecular subtype. We established a lentiCre-induced Kras and $L k b 1$ mutant genetically engineered mouse model ( $\left.K L_{\text {Lenti }}\right)$ that develops $100 \%$ lung adenocarcinoma and showed that high spatiotemporal FAK activation occurs in collective invasive cells that are surrounded by high levels of collagen. Modeling invasion in 3D, loss of $L k b 1$, but not $p 53$, was sufficient to drive collective invasion and collagen alignment that was highly sensitive to FAK inhibition. Treatment of early, stage-matched $K L_{\text {Lenti }}$ tumors with FAK inhibitor monotherapy resulted in a striking effect on tumor progression, invasion, and tumor-associated collagen. Chronic treatment extended survival and impeded local lymph node spread. Lastly, we identified focally upregulated FAK and collagen-associated collective invasion in KRAS and LKB1 comutated human lung adenocarcinoma patients. Our results suggest that patients with $L K B 1$ mutant tumors should be stratified for early treatment with FAK inhibitors.

Conflict of interest: The authors have declared that no conflict of interest exists.

Submitted: September 2, 2016 Accepted: January 26, 2017 Published: March 9, 2017

Reference information: JCI Insight. 2017;2(5):e90487. https:// doi.org/10.1172/jici.nsight.90487.

\section{Introduction}

$L K B 1$ ( $S T K 11)$ is the second most frequently mutated tumor suppressor gene in human lung adenocarcinoma, after TP53, and it is inactivated in up to $30 \%$ of $K R A S$ mutant non-small cell lung cancers (NSCLC) (1-5). In mouse models, loss of $L k b 1$ in vivo promotes the aggressive, metastatic spread of Kras mutant lung cancer and renders tumors largely resistant to both docetaxel monotherapy and to combination therapy with a MEK inhibitor $(6,7)$. Although cytotoxic and cytostatic therapies are in development to target $L K B 1$ mutant vulnerabilities (8-11), a comprehensive therapeutic strategy designed to target the aggressive biology of KRAS and $L K B 1$ mutant tumors has yet to lead to a clinical intervention. Immune checkpoint therapies have shown promise in the treatment of NSCLC, yet recent work has identified distinct molecular features of $K R A S$ and $L K B 1$ mutant tumors, which include suppression of immunoediting and resistance to PD-1-targeting antibodies (12-14). Hence, there is an urgent need for targeted therapies against KRAS and LKB1 mutant NSCLC.

We and others have identified LKB1 as a negative regulator of the focal adhesion kinase/Src (FAK/Src) signaling pathway in tumor cells $(11,15-17)$. FAK is a nonreceptor tyrosine kinase that serves as a master regulator of cell adhesion during cell motility and has kinase-dependent roles in cell invasion (18). As such, several anticancer agents targeting FAK activity are in clinical development (19).

In our study, we take a multidisciplinary approach to establish FAK as a therapeutic vulnerability in $L K B 1$ mutant lung adenocarcinoma in vivo. We have established a lentiviral-Cre induced Kras and $L k b 1$ 
model of NSCLC that, unlike previous models, develops 100\% lung adenocarcinomas. We used this model to design the first rolling-enrollment, stage-matched preclinical trial targeting adhesion signaling, with the goal of inhibiting the invasive progression of KRAS and $L K B 1$ mutant lung tumors in a clinical setting. Our data show that FAK is hyperactive in dense, collagen-associated collective invasion packs (CIPs) in late-stage $K R A S$ and $L K B 1$ mutant mouse and human tumors and that treating mice at early stages with a FAK inhibitor results in pleiotropic therapeutic responses, including a reduction in tumor burden and invasiveness, and a decrease in the tumor-associated collagen network. Our data support the implementation of early personalized therapy targeting FAK in LKB1-inactivated cancers and establish a rationale for future combination treatment strategies.

\section{Results}

A lenti-Cre Kras ${ }^{G 12 D}$ Lkb $^{\text {fllf }}$ lung adenocarcinoma model reveals FAK is highly active in collagen-associated CIPs in vivo. We created a lentiviral-Cre-induced (lenti-Cre-induced) $L S L-K r a s^{G 12 D /+} L k b 1^{f l / l}$ mouse model $\left(K L_{\text {Lenti }}\right)$ (Figure 1A). Unlike previous adeno-Cre models, $K L_{\text {Lenti }}$ mice develop $100 \%$ lung adenocarcinomas $(n=93)$, the predominant subtype of human NSCLC that harbors $L K B 1$ loss (5). Tumors from $K L_{\text {Lenti }}$ mice exhibit the major histologic subtypes of lung adenocarcinoma, including acinar, lepidic, papillary, and solid (mucinous) and develop metastases to the mediastinal lymph nodes $(52 \%, n=33)$ (Supplemental Figure 1A; supplemental material available online with this article; https://doi.org/10.1172/jci.insight.90487DS1). In order to gain spatial information on FAK activity in primary Kras and $L k b 1$ lung adenocarcinoma in vivo, we used IHC to assess FAK autophosphorylation at site 397 (pYFAK ${ }^{397}$ ) in early- and late-stage $K L_{\text {Lenti }}$ primary tumors. Lung adenocarcinoma in situ (AIS) expressed levels of pYFAK ${ }^{397}$ that were similar to the nontransformed cells in the alveolar space (Figure 1C). In contrast, invasive adenocarcinomas expressed high levels of pYFAK, with peak levels detected in a subset of cells at the invasive front of tumors and in cell clusters surrounded by stroma (Figure 1C). We refer to these cells as CIPs. To test the specificity of this phenotype to $L k b 1$ loss, we performed IHC to pYFAK ${ }^{397}$ on early- and late-stage $\operatorname{Kras}^{G 12 D} p 53^{\text {fl/fl}}$ mouse lung adenocarcinoma $(K P)$ but only detected high levels of pYFAK in immune cells (Supplemental Figure 1B). To confirm that Lkb1 mutant $\mathrm{pYFAK}^{+}$cells were derived from collective adenocarcinoma cells, we confirmed that CIPs express surfactant protein C (SP-C) and maintain junctional E-Cadherin (E-Cad) (Figure 1B and Supplemental Figure 2A).

Collective migration in breast cancer leads to collagen remodeling, which facilitates local invasion $(20,21)$. We imaged $K L_{\text {Lenti }}$ tumors at early and late stages using second harmonic generation (SHG) to detect collagen. We identified low levels of unaligned collagen in early-stage tumors (Figure 1, D and E). In contrast, we detected high levels of aligned collagen in $K L_{\text {Lenti }}$ invasive tumors. Aligned collagen fibers (perpendicular to the axis of invasion) were identified in close approximation to CIPs (Figure 1D). We performed a similar analysis in $K P$ invasive lung tumors, which revealed a low level of unaligned collagen (Figure $1 \mathrm{E}$ and Supplemental Figure 1C). Taken together, our data suggest that autonomous upregulation of FAK activity in tumor cells accompanies collagen remodeling and collective invasion, and is unique to the Kras and $L k b 1$ molecular subtype of lung adenocarcinoma in vivo.

$L k b 1$ is required to restrict FAK-dependent collective invasion and collagen alignment in $3 D$ tumor spheroids. To investigate cell biologic and functional requirements for FAK signaling in Kras and Lkb1 mutant tumor cells, we tested invasion phenotypes using 3D spheroid assays in $\operatorname{Kras}^{G 12 D} p 53^{\text {fl/fl}}\left(\mathrm{Lkb} 1 \mathrm{WT}\right.$ ) and $\mathrm{Kras}^{G 12 D} p 53^{\text {fl/fl}}$ $L_{k b} 1^{f l / f l}$ (Lkb1-null) mouse tumor cell lines (8). Western blot analysis confirmed defective Lkb1 pathway signaling in Lkb1-null cells (Supplemental Figure 3A). Lkb1-null spheroids invaded into collagen as a collective sheet of cells (Figure 2A and Supplemental Video 1), similar to the collective invasion we observed in vivo. Conversely, Lkb1 WT spheroids exhibited single-cell invasion (Figure 2A and Supplemental Video 2). Molecular analysis confirmed that invasive Lkb1-null cells maintain E-Cad in clusters of cells at the leading edge in contrast to Lkb1 WT spheroids that lack E-Cad and express Vimentin (Supplemental Figure 2, C and D). Analysis of pYFAK showed an accumulation or stabilization of pYFAK ${ }^{397}$ sites at the leading edge of collectively invading Lkb1-null cells that is not observed in Lkb1 WT cells (Figure 2A and Supplemental Figure 2B). Treatment of Lkb1-null spheroids with the FAK inhibitors PF-562271 and GSK2256098 (GSK6098) (19) lead to a downregulation of FAK activity (Supplemental Figure 3, B-D) and a significant suppression of Lkb1-null collective invasion (as measured by invasive area) into collagen (Figure 2, B and $\mathrm{C}$ and Supplemental Figure 3E). In contrast, inhibition of FAK activity in Lkb1 WT cells did not exhibit a significant reduction in invasion. We conclude that Lkb1 mutant lung tumor cells are uniquely reliant on hyperactive FAK signaling during collective invasion into collagen. 
A

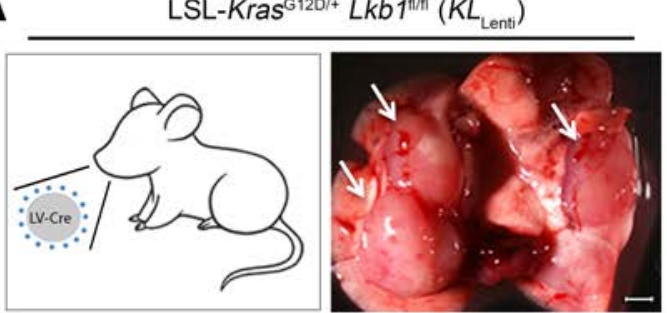

C
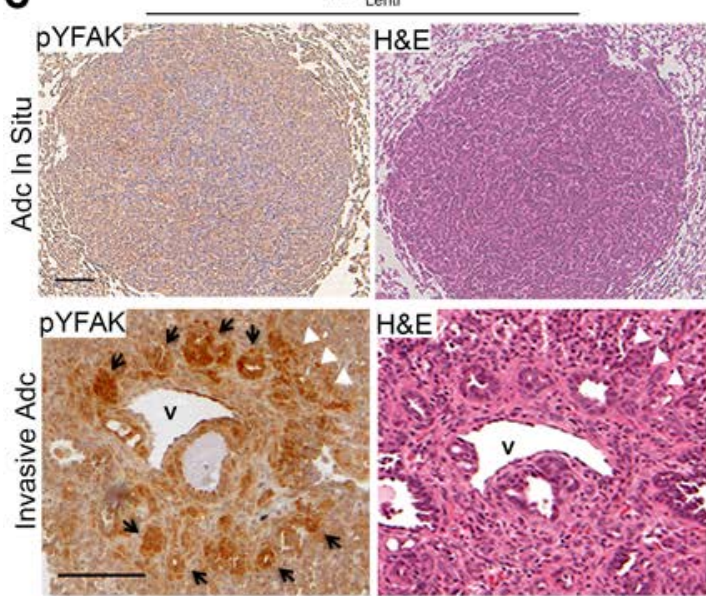

E

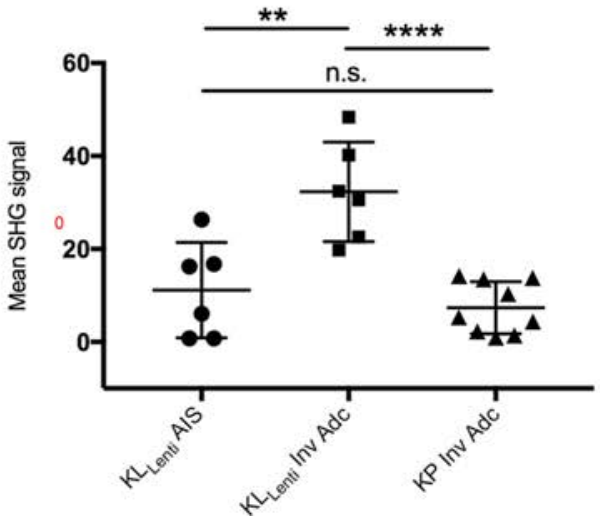

B

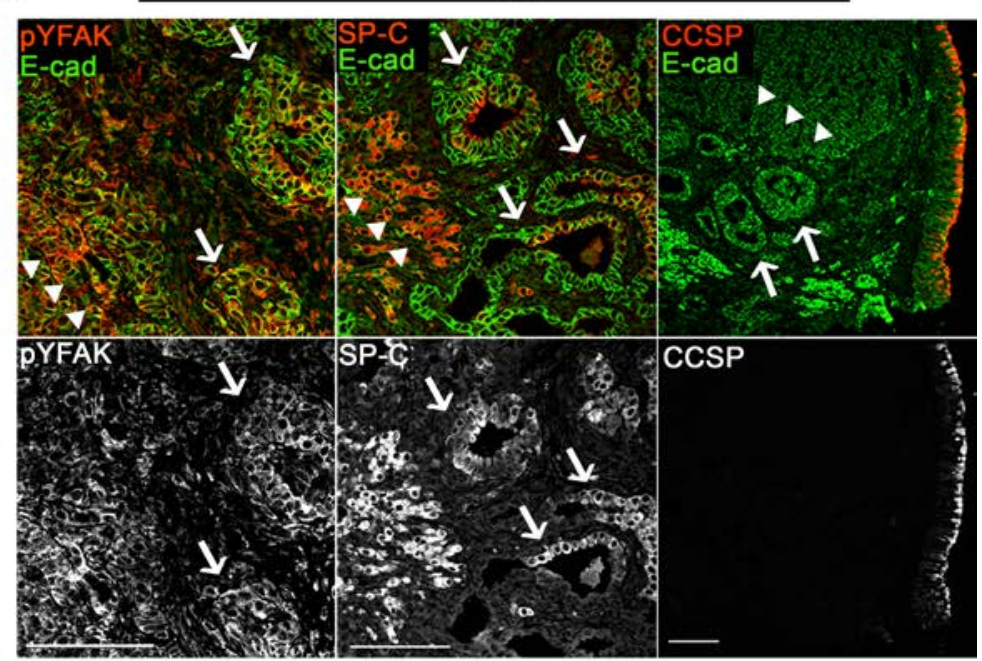

D

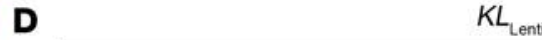

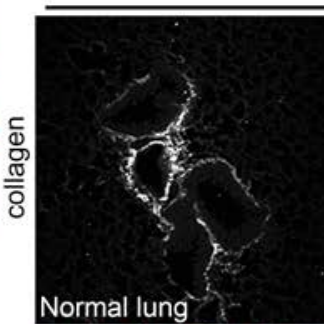
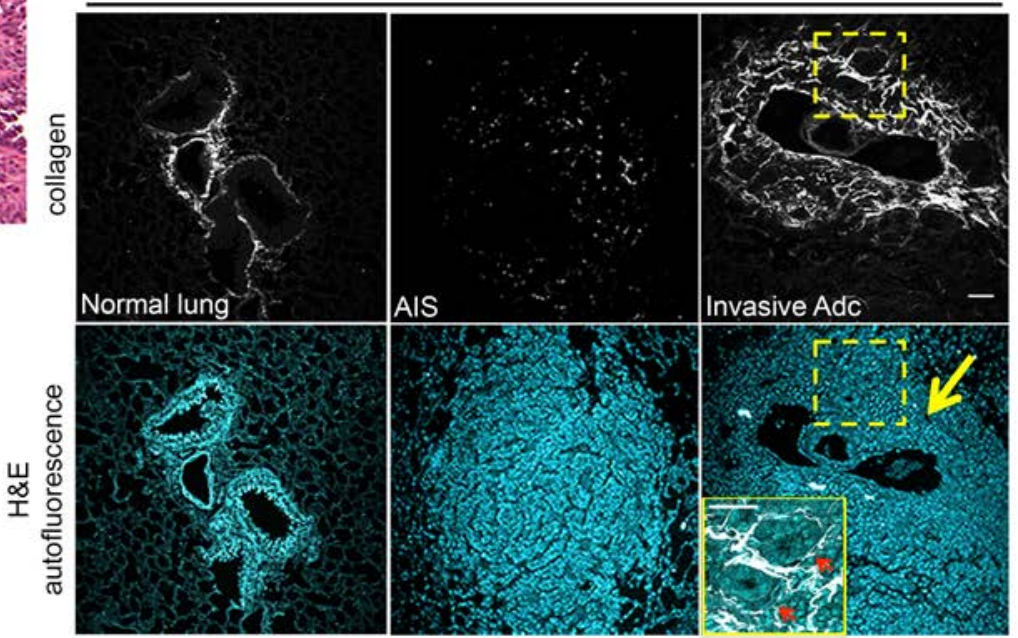

Figure 1. A lenti-Cre $\mathrm{Kras}^{\mathrm{G} 120} \mathrm{Lkb}^{\mathrm{fl} / \mathrm{fl}}$ mouse model ( $\mathrm{KL}_{\mathrm{Lenti}}$ ) of lung adenocarcinoma reveals high levels of active FAK in collagen-associated collective invasion packs (CIPs) in vivo. (A) Lenti-Cre model of $K \mathrm{ras}^{\mathrm{G}}{ }^{120} \mathrm{Lkb} \mathrm{f}^{\mathrm{fl} / \mathrm{f}}$ lung adenocarcinoma. White arrows indicate primary lung tumors. Scale bar: $50 \mathrm{~mm}$. (B) Double immunofluorescence analysis of pYFAK ${ }^{397}$ /E-Cad (left column), E-Cad/SP-C (middle column), and E-Cad/CCSP (right column) from serial sections of late-stage $K L_{\text {Lenti }}$ invasive primary tumors. Arrows mark CIPs, and arrowheads are located behind the invasive front. Scale bars: 100 $\mu \mathrm{m}$. (C) Representative images of IHC of PYFAK ${ }^{397}$ and serial H\&E-stained sections from $K L_{\text {Lenti }}$ adenocarcinoma in situ (AIS) (top) and invasive adenocarcinoma (Inv Adc) (bottom) $(n=6)$. Black arrows mark pYFAK-positive invasive tumor cells, and arrowheads are located behind the invasive front. Scale bars: $200 \mu \mathrm{m}$. v, vessel. (D) SHG (white; top row) imaging of collagen in $K L_{\text {Lenti }}$ Inv Adc compared with a normal bronchovascular bundle and AIS. Cell structure is visualized by H\&E autofluorescence (pseudo-colored blue). Scale bar: $50 \mu \mathrm{m}$. Yellow dashed boxes outline regions merged and magnified in inset. Scale bar: $50 \mu \mathrm{m}$. Red arrows mark CIPs, and yellow arrow marks direction of invasive front. (E) Quantitation of collagen density by mean SHG signal intensity from lung tumor sections of the indicated genotype and stage ( $n=2$ lung lobes each from 3 independent mice $\left[K L_{\text {Lenti }}\right.$ AIS and $\left.K L_{\text {Lenti }} I n v A d c\right]$, and $n=1-2$ lung lobes from 6 independent mice [KP Inv Adc]). Data are represented as mean \pm SD. $P$ values were calculated using 1-way ANOVA with Tukey's multiple comparisons test. ${ }^{* * *} P<0.0001 ;{ }^{* *} P<0.01$.

We next examined whether collagen alignment was FAK dependent during Lkb1 WT and Lkb1null spheroid invasion using SHG. During invasion, Lkb1-null spheroids exhibit increased collagen alignment compared with LKB1 WT spheroids (Figure 2, D and E). We tested the requirement for FAK activity by treating Lkb1-null spheroids with the FAK inhibitor PF-562271 and implemented a local alignment coefficient to quantify the effect (Figure 2, E and F) (18). Our results indicate that Lkb1-null spheroids are indeed dependent on FAK activity to align collagen fibers during collective 3D invasion. 
A

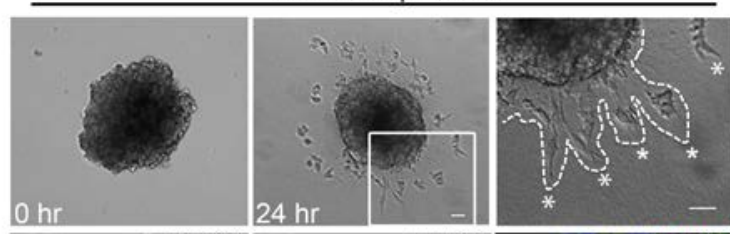

Lkb1 WT spheroids

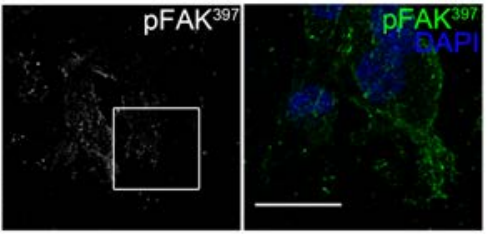

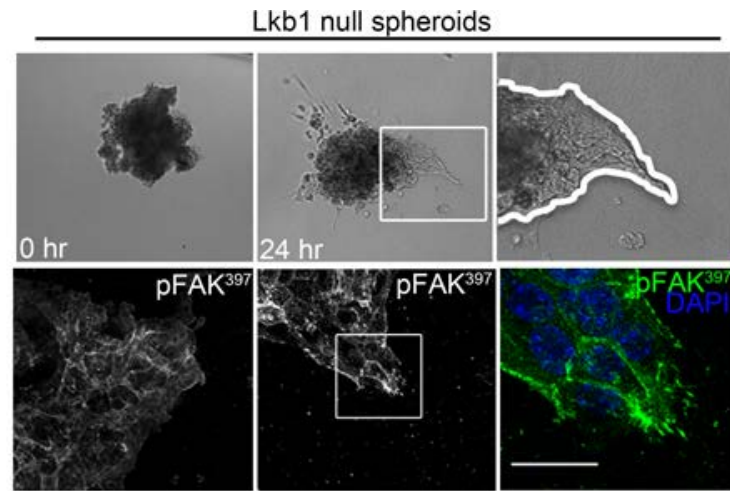

C
B

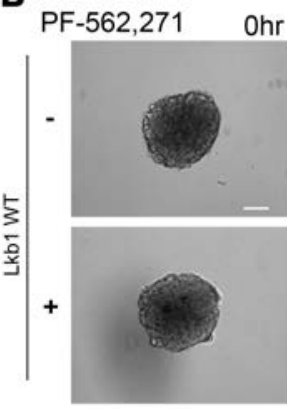

D

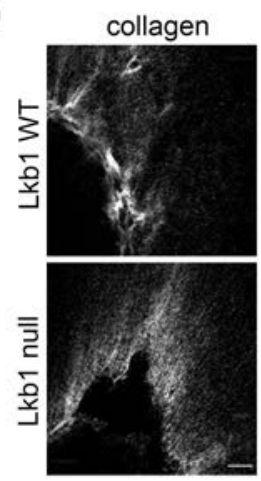

$50 \mathrm{hr}$
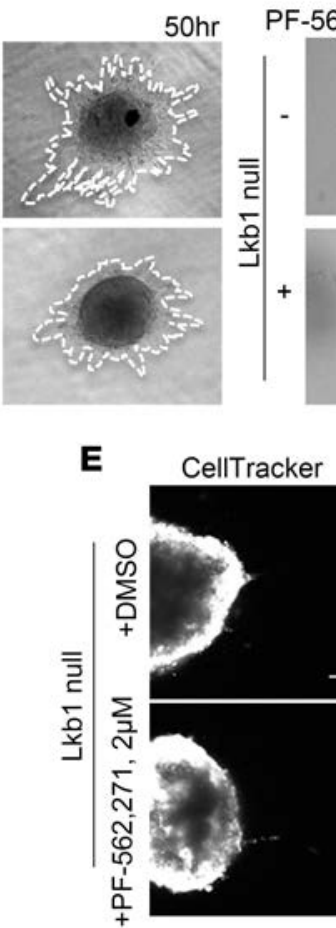

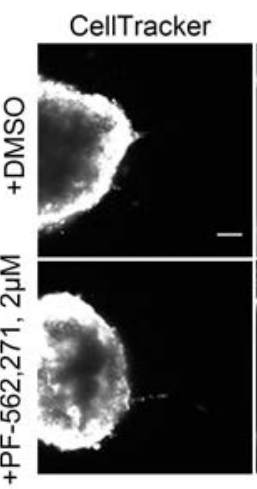

62,271

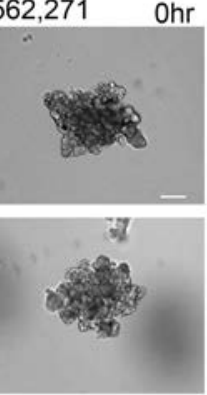

Or
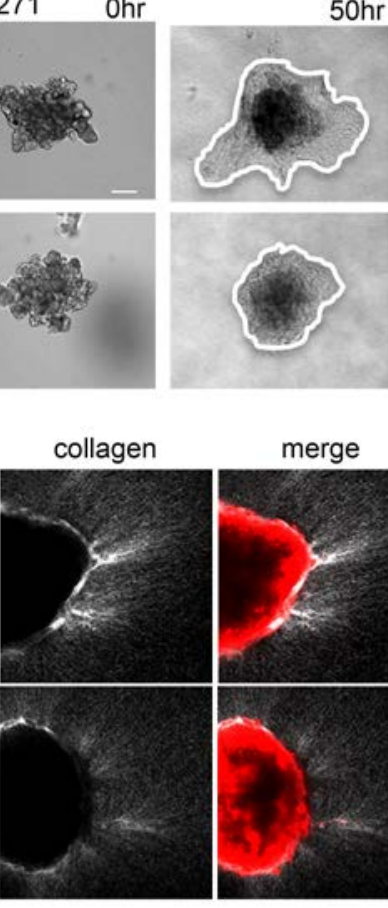

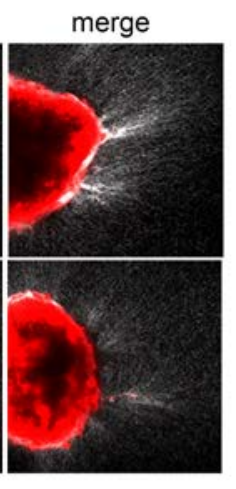

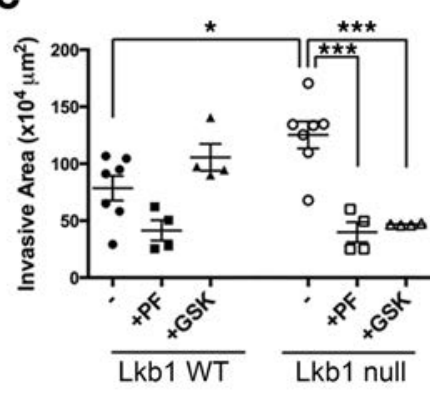

$\mathbf{F}$

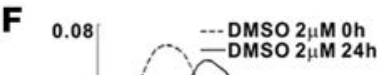

Figure 2. Lkb1 mutant tumor cells exhibit collective, FAK-dependent 3D cell invasion and collagen remodeling. (A) (Top) Representative brightfield images of Lkb1 WT and Lkb1-null mouse tumor cell spheroids that were embedded in a collagen I matrix and allowed to invade for 24 hours. Area in white boxes is magnified to the right. Dashed line with asterisks marks single invasive cells, and sold line marks collectively invading cells. Scale bars: $50 \mu \mathrm{m}$. (Bottom) Representative immunofluorescence images of pYFAK ${ }^{397}$ at the invasive front in Lkb1 WT and Lkb1-null spheroids after 48 hours of invasion into a collagen matrix. Area in white boxes is magnified to the right. Scale bars: $50 \mu \mathrm{m}$. (B) Representative images of spheroids formed from Lkb1 WT and Lkb1-null cells analyzed for total invasion into a collagen matrix in the presence of FAK inhibitor PF-562271 at 250 nM. DMSO was used as a vehicle control. Scale bars: $100 \mu \mathrm{m}$. (C) Quantification of spheroid invasion after treatment with either PF-562271 at $250 \mathrm{nM}$ or GSK6098 at $2 \mu \mathrm{M} . n=4$ spheroids (FAK inhibitors) or $n=7$ spheroids (DMSO). Data are represented as mean \pm SD. $P$ values were calculated using 1-way ANOVA with Tukey's multiple comparisons test, ${ }^{*} P<0.05^{* *}, P<0.0001$. (D) Second harmonic generation imaging of collagen after invasion by $3 D$ tumor cell spheroids of the indicated genotypes. Scale bar: $50 \mu \mathrm{m}$. (E) Lkb1-null 3D tumor spheroids treated with vehicle (DMSO, top panels) or PF-562271 (bottom panels) embedded in a collagen matrix and visualized 24 hours later by CellTracker (tumor cell spheroids, red in merge) and second harmonic generation (SHG) to visualize collagen fibers. Scale bar: $50 \mu \mathrm{m}$. (F) Collagen alignment coefficient of DMSO and PF-562271-treated Lkb1-null tumor cell spheroids shown in $\mathbf{E}$.

Early treatment of Kras and Lkb1 mutant lung tumors with a FAK inhibitor suppresses tumor progression in vivo. Based on our previous results, we hypothesized that a dependence on high FAK activity could represent a vulnerability in Kras and $L k b 1$ lung adenocarcinoma. To test this in vivo, and to treat stage-matched early tumors, we combined a Rosa26-luciferase allele with our $K L_{\text {Lenti }}$ mouse to create $K L L_{\text {Lenti }}$ mice. Total flux in $K L L_{\text {Lenti }}$ mice is proportional to tumor grade and tumor burden, and it correlates with metastatic colonization of the lymph node (Figure 3A and Supplemental Figure 4), which allowed us to use photons/second (p/s) as a 
A

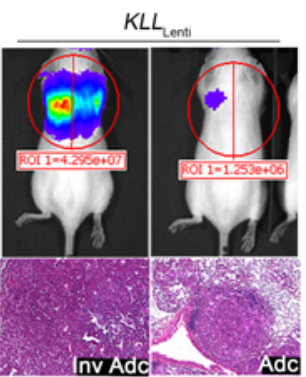

E

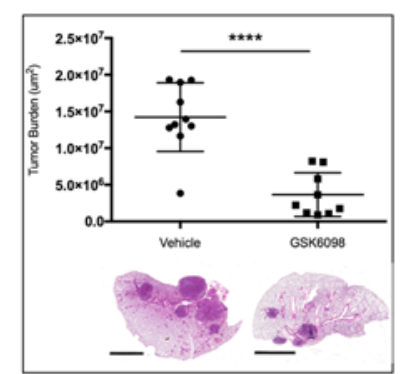

B

Rolling Enrollment Trial Design

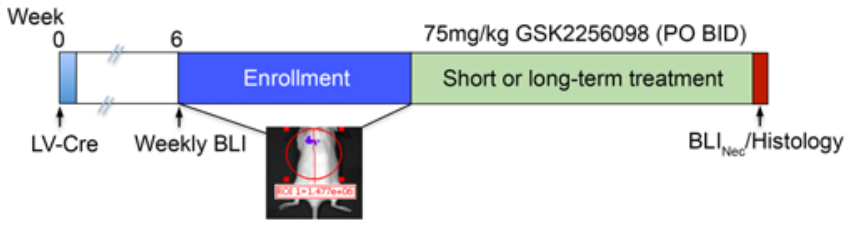

C
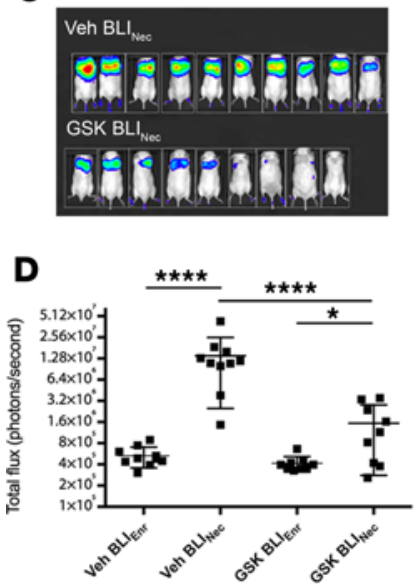

$\mathbf{F}$

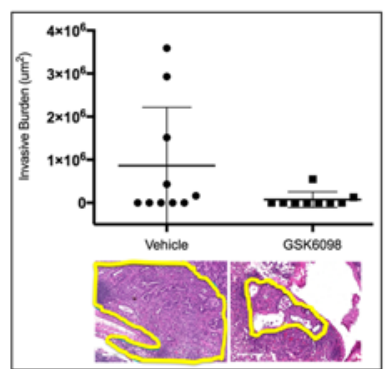

G

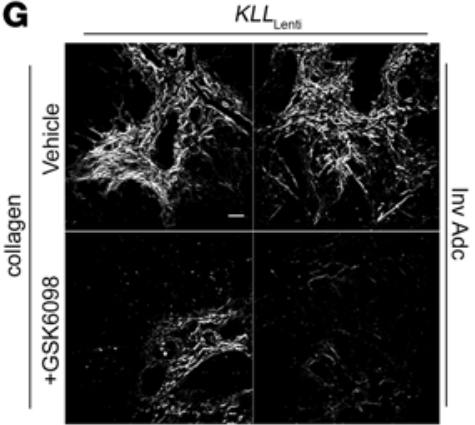

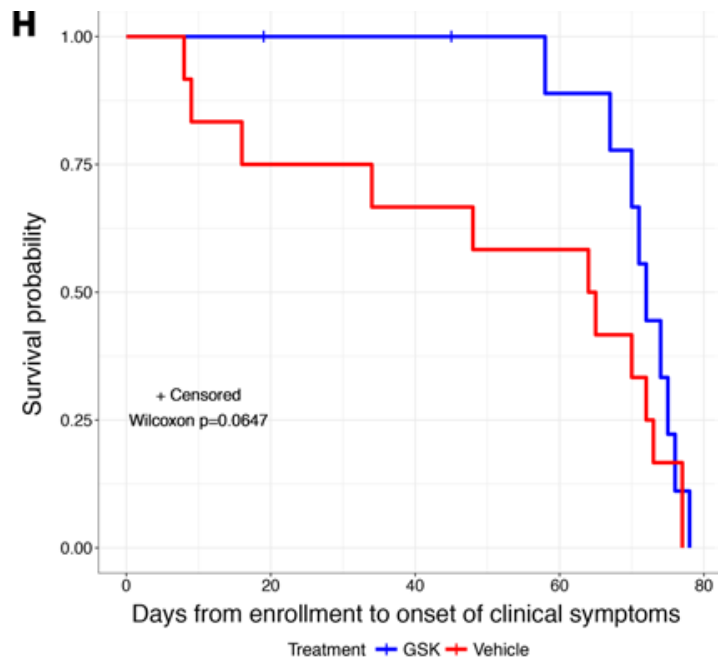

Treatment + GSK + Vehicle
I

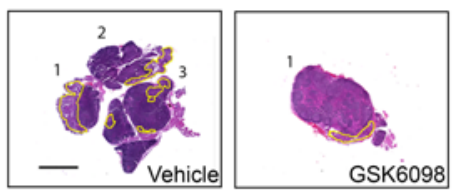

J

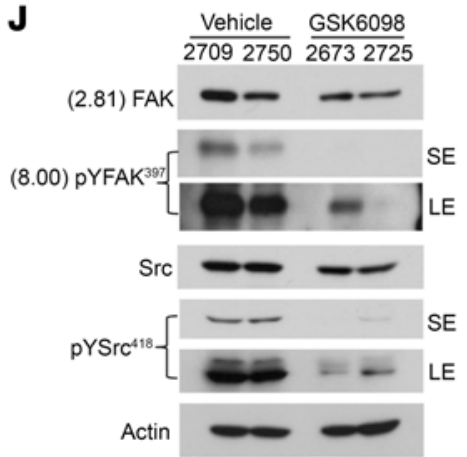

Figure 3. Treatment with a FAK inhibitor results in multiple therapeutic responses in Kras and Lkb1 mutant lung adenocarcinoma in vivo. (A) (Top panel) Dorsal

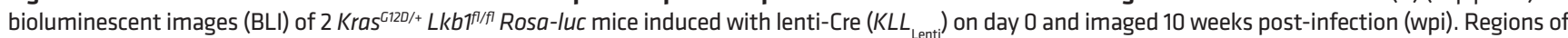
interest (ROIs) indicate total flux in photons/second. (Bottom panel) H\&E-stained primary lung tumors from mice pictured above (50x). (B) Graphic of preclinical trial design. (C) BLI images of vehicle- and GSK6098-treated mice after 5-week treatment protocol (Nec, necropsy). (D) Total flux (photons/second) at enrollment (Enr) vs. necropsy (Nec) for vehicle- $(n=10)$ and GSK6098-treated mice $(n=9)$ in 5-week treatment trial. Each box represents an individual mouse pictured in C; one-way ANOVA with Tukey's multiple comparisons test, $\left.{ }^{* * *} P<0.0001,{ }^{*} P<0.05\right)$. (E) Mean tumor burden $\left(\mu m^{2}\right)$ in vehicle- $(n=10)$ vs. GSK6098-treated mice $(n=9) ; 2$-tailed $t$ test, ${ }^{* * * *} P<0.0001$. (Bottom panel) Representative images of lung tumors in vehicle- vs. GSK6098-treated mice. Scale bar: 3 mm. (F) Mean invasive burden in vehicle- ( $n=10)$ vs. GSK6098-treated mice $(n=9)$; 2 -tailed $t$ test, $P=0.1089$. (Bottom panel) Representative images of invasive tumors (outlined in yellow) in vehicle- vs. GSK6098-treated mice (40x; Nanozoomer; 8x digital zoom). (G) SHG imaging of collagen (white) in vehicle- vs. GSK6098-treated $K L L_{\text {Lenti }}$ lung tumors. Images are 2 independent stage-matched (Inv Adc) fields per treatment group. (H) Kaplan Meier analysis of days from enrollment to the onset of clinical symptoms of chronically treated vehicle $(n=12)$ vs. GSK6098 $(n=11) K L L_{\text {Lenti }}$ mice. Wilcoxon test, $P=0.0647$. (I) Representative lymph nodes from chronically treated vehicle (3 independent lymph nodes from a single mouse pictured [numbered 1-3] vs. GSK6098 [1 positive lymph node from a single mouse pictured]). Yellow outlines mark metastatic tumor cells. Scale bar: $2 \mathrm{~mm}$. (J) Western blot analysis of the FAK/Src pathway in vehicle- vs. GSK6098-treated lung lobes. Numbers in parentheses indicate fold change in the vehicle/GSK6098 mean signal intensity ratio. SE, short exposure; LE, long exposure.

sensitive measure of invasive progression and metastatic potential. To facilitate the translation of our results into human trials, mice were treated with GSK6098, an ATP-competitive FAK inhibitor that has shown efficacy in animal models and is currently in clinical development (19). Western blot analyses indicated that GSK6098 inhibited FAK pathway activity in Kras and Lkb1 mutant mouse lung tissue in vivo (Figure $3 J)$. To ensure enrollment of mice with early-stage (preinvasive) tumors, a test cohort of $K L L_{\text {Lenti }}$ mice were 
bioluminescent imaged to establish a maximum enrollment score $\left(\mathrm{BLI}_{\mathrm{Enr}}\right)$. For the trial, $K L L_{\text {Lenti }}$ mice were imaged until luciferase-positive tumors were detected at or below $\mathrm{BLI}_{\mathrm{Enr}}$, after which they were randomly assigned to receive either vehicle $(n=10)$ or GSK6098 $(n=9)(75 \mathrm{mg} / \mathrm{kg}$ PO BID) (Figure 3B and Supplemental Table 2). Mice were treated for 5 weeks, reimaged $\left(\mathrm{BLI}_{\mathrm{Nec}}\right)$ and necropsied for histology. Five-week treatment with GSK6098 had a significant effect on tumor progression in $K L L_{\text {Lenti }}$ mice, as measured by total flux (Figure 3, C and D). Moreover, histologic staging of tumor grade revealed a correlation between GSK6098 treatment and lower-grade tumors (Supplemental Table 1). Strikingly, $K L L_{\text {Lenti }}$ mice showed significant reductions in lung tumor burden after treatment with GSK6098 (Figure 3E). No changes in caspase-dependent apoptosis nor cell proliferation were observed in GSK6098-treated tumors (Melissa Gilbert-Ross, unpublished observations); however, a positive trend in the number of tumor-associated CD45 $5^{+}$leukocytes was observed in GSK6098-treated mice (Supplemental Figure 4D). This suggests that FAK inhibition may prevent or delay tumor onset and/or progression via effects on the tumor immune microenvironment. We quantitated the effect of GSK6098 treatment on invasion by measuring the area of invasive tumors in vehicle- vs. GSK6098-treated mice (invasive burden). GSK6098-treated mice exhibited a strong trend in decreased invasive burden (Figure 3F). No correlation was found between mean total tumor burden and invasive burden in control mice (Supplemental Figure 4C). This suggests that the inhibition of invasion in GSK6098-treated mice is not due solely to a decrease in tumor burden. Similar to our in vitro results, 5 -week treatment of $K L L_{\text {Lenti }}$ mice with the FAK inhibitor GSK6098 led to a decrease in the levels and alignment of collagen in stage-matched grade IV vehicle- vs. GSK6098-treated lung tumors (Figure 3G). Taken together, our data support our hypothesis that FAK activity represents a vulnerability in Kras and $L k b 1$ mutant lung adenocarcinoma in vivo, with treatment resulting in both tumor cell-autonomous and tumor microenvironmental effects.

We further evaluated whether chronic treatment with GSK6098 could extend the time to onset of clinical symptoms in mice with Kras and $L k b 1$ mutant lung adenocarcinoma. $K L L_{\text {Lenti }}$ mice were imaged until tumor onset and randomly assigned to either vehicle or GSK6098 treatment ( $75 \mathrm{mg} / \mathrm{kg}$ PO BID) to be continued until mice developed clinical symptoms (see Methods). To account for the emergence of potential resistance mechanisms during long-term treatment, survival curves were analyzed using the Wilcoxon test. Taking into account the relatively small sample size, the analysis indicates a significant difference in days to onset of clinical symptoms in GSK6098- vs. vehicle-treated $K L L_{\text {Lenti }}$ mice (Figure 3H). Analysis of the mediastinal lymph nodes from these mice revealed a significant association between treatment and the extent to which metastatic tumor cells spread to 2 or more lymph nodes (vehicle $=5 / 7$ vs. GSK6098 $=0 / 5$; Fisher's exact test, $P=.0278$ ) (Figure 3I).

$K R A S$ and LKB1 comutation in human lung adenocarcinoma patients is associated with focal $p Y F A K$ upregulation and collagen-associated CIPs. We investigated whether our results could be applied to identify KRAS and $L K B 1$ mutant lung adenocarcinoma patients who may benefit from FAK pathway inhibition. We used a targeted and clinically validated next-generation sequencing (NGS) mutation panel (22) to assay formalin-fixed, paraffin-embedded (FFPE) patient tissue to identify $K R A S$ and $K R A S$ and $L K B 1$ mutant lung adenocarcinoma. Tissue sections from 12 total patients ( $n=6 \mathrm{KRAS}$ and $n=6 \mathrm{KRAS}$ and $L K B 1$ ) were assayed by confocal microscopy for E-Cad expression, and identical fields were then imaged via SHG to visualize collagen. Collagen density was then quantified by measuring mean SHG signal intensity across the entire field of view ( 2 fields/patient). In KRAS-only (LKB1 WT) lung adenocarcinoma, we identified E-Cad in noninvasive tumor cells that was associated with low levels of collagen (Figure 4, A and C). In contrast, in KRAS and $L K B 1$ mutant lung adenocarcinoma, we identified E-Cad ${ }^{+} \mathrm{CIPs}_{\text {shat were }}$ associated with high levels of collagen (Figure 4, B and C). Importantly, E-Cad ${ }^{+}$CIPs had high levels of pYFAK $^{397}$ (Figure 4B). Next, we sought to determine if we could identify a pattern of pYFAK ${ }^{397}$ upregulation in comutated vs. KRAS-only mutant patients of various stages. We performed IHC for $\mathrm{pYFAK}^{397}$ on 13 patient samples ( $n=7 K R A S$ and $n=6 K R A S$ LKB1 patients), and tumor tissue was scored as either having a majority diffuse pYFAK staining pattern or alternatively as having focally upregulated pYFAK (see Methods). Using this criteria, pYFAK focal upregulation was significantly associated with $K R A S$ and LKB1 comutated patients (Figure 4D and Supplemental Table 3).

\section{Discussion}

LKB1 is the third most frequently altered gene in adenocarcinoma of the lung and leads to aggressive metastatic disease in multiple tumor types. Currently, there are no targeted therapies against tumors with this genetic alteration. Our study presents a lentiviral-Cre-induced Kras ${ }^{G 12 D} L k b 1$ mutant genetically engineered mouse model (GEMM) that results in the exclusive generation of lung adenocarcinoma. We used 
A

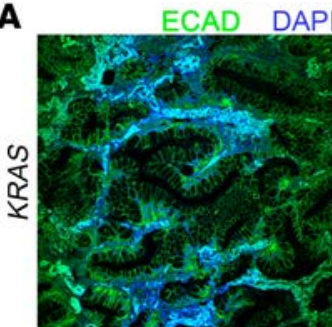

B
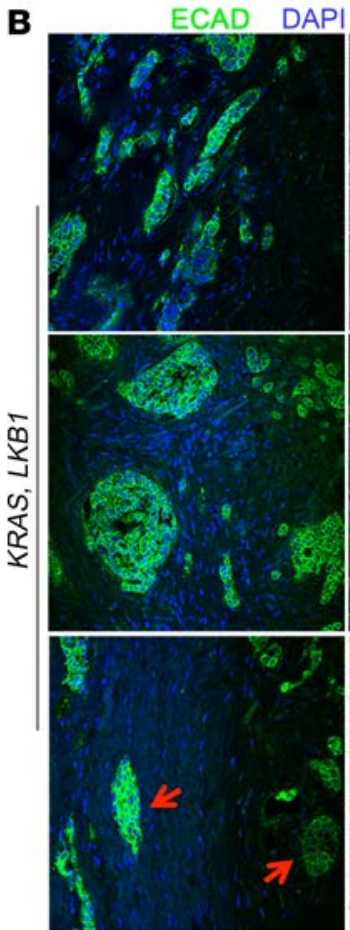

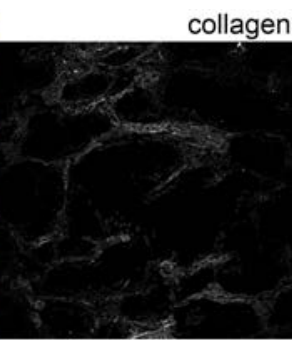

collagen

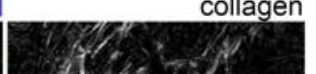

C

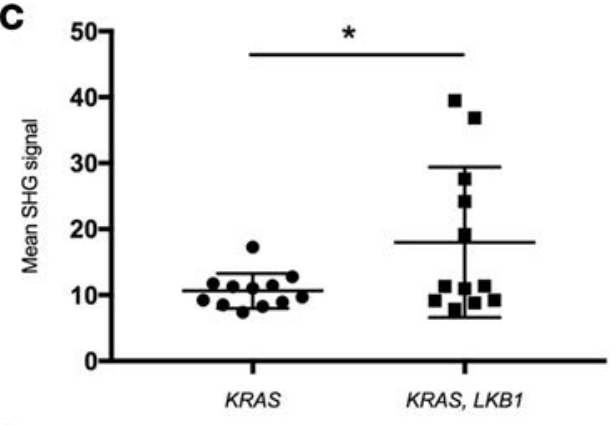

D

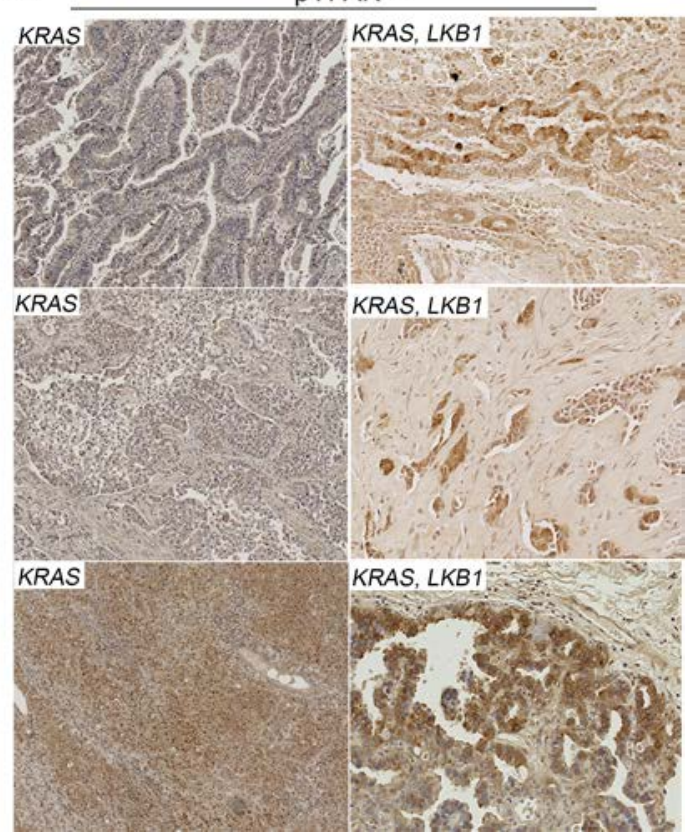

Figure 4. KRAS and LKB1 comutated human lung adenocarcinoma exhibits focal upregulation of pYFAK and collagen-associated collective invasion packs (CIPs). (A) Representative confocal image (left) of E-Cadherin (green) and SHG image (right) of collagen (white) in human KRAS mutant lung adenocarcinoma. The field imaged by confocal microscopy was subsequently reimaged for collagen using SHG (200x) (B) Representative confocal images (left column) and SHG images of E-Cadherin (green) and collagen (white) of 2 independent fields from a KRAS and LKB1 mutant human primary lung adenocarcinoma (top and middle panels). As in $\mathbf{A}$, fields imaged by confocal microscopy were subsequently reimaged with SHG to detect collagen. Confocal imaging of E-Cadherin (left) and an adjacent section stained for pYFAK (right) is pictured in the bottom panel. Red arrows mark CIPs present in both sections that are E-Cadherin- and pYFAK-positive. All panels imaged at 200x. (C) Quantitation of collagen density by mean SHC signal intensity. Each data point represents an independent field, and 2 independent fields were imaged per patient with $n=6 K R A S$ and $n=6 K R A S$ and $L K B 1$ mutant patients. Data are presented as mean \pm SD. Two-tailed $t$ test; ${ }^{*} P<$ 0.05. (D) Representative images of pYFAK ${ }^{397} I H C$ from 3 independent patient samples per indicated genotype (representative images from a total of $n=7 K R A S$ and $n=6 K R A S$ and $L K B 1$ mutant patients samples [15 total patients]).

our model to conduct the first rolling-enrollment preclinical trial and to show that adhesion therapy with a FAK inhibitor is a viable treatment strategy for this high-risk molecular subtype.

Evidence from mouse models indicates that Kras and Lkb1 mutant lung adenocarcinoma is largely resistant to both standard-of-care docetaxel and combination treatment with a MEK inhibitor (7). This is in contrast to both Kras and Kras, p53 mutant lung cancer models. Clearly, the molecular heterogeneity of coexisting tumor suppressor mutations effects therapeutic response. Our results suggest that the Kras and Lkb1 molecular subtype is highly sensitive to FAK inhibition in vivo due to deregulated pathway signaling during collective invasion and collagen alignment, and they predict that adhesion therapy could represent the first viable targeted-treatment strategy for these patients. Our results do not rule out the possibility that additional molecular subtypes will also benefit from FAK inhibitors. Also, it remains to be addressed whether the full spectrum of $L K B 1$ loss-of-function mutations found in human tumors, 
in addition to patients with functional inactivation of the LKB1-AMPK axis in LKB1 mutation-negative tumors (13), will benefit from FAK therapy.

Importantly, our data supports the design of a clinical trial in early-stage $K R A S$ and $L K B 1$ mutant lung adenocarcinoma patients and suggest that current clinical trials designed to enroll patients with metastatic or recurrent disease may not be beneficial. Early clinical intervention with FAK inhibitors creates the need to compare and/or combine adhesion therapy with standard-of-care agents. We propose that FAK inhibitors may synergize with standard agents and targeted therapies, and may render KRAS and LKB1 mutant lung adenocarcinoma sensitive to immune checkpoint inhibition due to the increased access of immune cells to tumors afforded by the reduction in high levels of aligned collagen in the surrounding microenvironment. In conclusion, our results support the continued refinement of FAK inhibitors as a clinical strategy in selected lung adenocarcinoma patients and open a potential new therapeutic opportunity for additional tumor types with inactivated LKB1 and collective invasion strategies.

\section{Methods}

\section{Mouse Studies}

$L S L-K r a s^{G 12 D}$ (23) and $L k b 1^{f l / f l}$ (24) mice were obtained from the Mouse Models of Human Cancers Consortium to create $K L_{\text {Lenti }}$ mice. Rosa26-Lox-Stop-Lox-Luc mice were obtained from Jackson Laboratories courtesy of Bill Kaelin (25) to create $K L L_{\text {Lenti }}$ mice. $K P$ lung tumor sections were obtained from AL Kasinski (Purdue University, West Lafayette, Indiana, USA). Lung tumors were induced by i.n. or intratracheal inhalation of lentiviral-Cre. $K L_{\text {Lenti }}$ mice exhibited decreased time to clinical symptoms and moribundity (16 weeks post infection [wpi]; $n=15$ ) when compared with $L S L-K r a s^{G 12 D /+}$ animals $(>25$ wpi; $n=5$ ). For the 5-week therapeutic trial, mice were imaged starting at 6 wpi at least once a week on an IVIS Spectrum (Perkin Elmer), enrolled under the maximum enrollment BLI $\left(1 \times 10^{6} \mathrm{p} / \mathrm{s}\right)$, and imaged again at the end of the 5-week treatment period $\left(\mathrm{BLI}_{\mathrm{Nec}}\right)$. Living Image software was used to compare longitudinally acquired calibrated signals. GSK6098 FAK inhibitor was delivered at $75 \mathrm{mg} / \mathrm{kg}$ PO BID for 5 weeks, after which mice were imaged and necropsied. For the chronic treatment trial, mice were imaged until tumor detection and then continuously treated with either vehicle or GSK6098 (75 mg/kg PO BID) until the onset of clinical symptoms (tachypnea, extreme weight loss), at which point treatment was stopped and the mice euthanized according to IACUC guidelines. Mouse lung tumors were scored by a board-certified lung pathologist using criteria previously published in ref. 26. Percent lymph node colonization was calculated as area occupied by metastatic tumor cells/total lymph node area $\left(\mathrm{um}^{2}\right)$. Lung tumor burden was calculated by measuring tumor area $\left(\mathrm{um}^{2}\right)$ area, and invasive burden was calculated by measuring the area of invasive tumors $\left(\mathrm{um}^{2}\right)$ using whole slide scanned images (40×; Olympus Nanozoomer) of H\&E-stained $K L L_{\text {Lenti }}$ lungs and lymph nodes using ImageScope software.

\section{Immunofluorescence/Immunohistochemistry}

Paraformaldehyde-fixed OCT-embedded mouse lung sections ( $5 \mu \mathrm{m}$-thick), and formalin-fixed paraffin-embedded mouse and human tumor lung sections were stained with rabbit anti-pYFAK ${ }^{397}$ (Abcam, catalog ab39967), rabbit anti-SP-C (Millipore, catalog AB3786), mouse anti-E-Cadherin (BD Biosciences, clone 36, catalog 610181), and rabbit anti-Vimentin (Cell Signaling Technology, clone R28, catalog 3932S). Secondary antibodies were purchased from ThermoFisher Scientific (anti-mouse Alexa Fluor 488; anti-rabbit Biotin-XX; Streptavidin, Alexa Fluor 555 Conjugate), and Vector Laboratories (Vector M.O.M and ImmPact reagents).

\section{D Invasion Assays}

Lkb1 WT and -null mouse tumor cells (Kwok-Kin Wong, Dana Farber Cancer Institute, Boston, Massachusetts, USA) were grown to $70 \%$ confluency and then trypsinized, neutralized, and resuspended in complete RPMI (Invitrogen). To generate spheroids, 3,000 cells were added to a Spheron Nunclon 96-well plate (ThermoFisher Scientific). After 3 days of incubation, spheroids were collected and resuspended in $2.0 \mathrm{mg} /$ $\mathrm{ml}$ Collagen Type I (Advanced Biomatrix), then plated in a Lab-Tek 8-well borosilicate bottom plate (ThermoFisher Scientific) for immunofluorescence or a 35-mm glass bottom dish (In Vitro Scientific) for multiphoton microscopy. After collagen solidified, complete RPMI was added to the top of the collagen matrix to provide a chemogradient for the spheroids. In 3D spheroid FAK inhibitor studies, $2 \mu \mathrm{M}$ PF-562271 was 
added directly to collagen before solidification and was also added to the growth media after solidification of the collagen. DMSO was used as a vehicle control. Spheroids were incubated at $37^{\circ} \mathrm{C}$ and $5 \% \mathrm{CO}_{2}$ for $24-48$ hours to allow for invasion. Spheroid invasion was quantified using ImageJ (NIH). The total area of cellular invasion into the collagen matrix was measured, and the spheroid bulk (i.e., the noninvasive area) was then subtracted from this total area, leaving an invasive area measurement.

\section{Spheroid microscopy}

Widefield imaging. Lkb1 WT and Lkb1-null spheroids were embedded in collagen as described above. Still images were taken at 0,24 , and 48 hours after embedding using an Olympus IX51 at 10× (0.30 numerical aperture [NA] air) and $20 \times(0.45 \mathrm{NA}$ air $)$.

Confocal imaging. Spheroids generated from Lkb1 WT and Lkb1-null cells were embedded in a 3D collagen matrix as previously described. Twenty-four hours later, cells were fixed using $4 \%$ paraformaldehyde (Electron Microscopy Sciences) for 15 minutes at room temperature and then quenched with $0.1 \mathrm{M}$ glycine in PBS (Sigma-Aldrich) for 10 minutes. Spheroids were then permeabilized with $0.5 \%$ Triton-X (Promega) for 1.5 hours, washed with PBS for 10 minutes, and blocked with 5\% normal goat serum (NGS, Jackson ImmunoResearch) for 1.5 hours. Spheroids were probed with rabbit $\mathrm{pFAK}^{\mathrm{Y} 397}$ (Abcam, catalog ab3996; 1:250 in PBS with 1\% BSA and 1\% NGS), rabbit anti-Vimentin (Cell Signaling Technology, clone R28, catalog 3932S), or mouse anti-E-Cadherin (BD Biosciences, clone 36, catalog 610181) overnight at $4^{\circ} \mathrm{C}$. Alexa Fluor 488 goat anti-rabbit (1:750 in PBS with 1\% NGS) or M.O.M. anti-mouse secondary was added the next day for 1.5 hours with gentle shaking at room temperature. Spheroids were then stained with 350 nM DAPI for 10 minutes, followed by 3 more PBS washes. Fixed spheroids were imaged using the FV1000 inverted confocal mounted on an Olympus IX81 inverted microscope (40×0.90 NA, Water PlanApo) with 1.3- $\mu \mathrm{m}$ z-stack intervals and sequential scanning (405 nm, $488 \mathrm{~nm}$ ). In FAK inhibitor-treated spheroids, PF-562271 (250nM) or DMSO control was added to the collagen matrix and growth media. Fresh growth media plus inhibitor was added every 10-12 hours. After 48 hours, cells were fixed and stained with pFAK $^{\mathrm{Y} 397}$ as described above.

\section{Multiphoton microscopy}

Spheroids of Lkb1 WT and Lkb1-null cells were imaged at 72 hours after embedding. In FAK inhibitor studies, spheroids were dyed using $1 \mu \mathrm{M}$ of Red CellTracker (Invitrogen) to visualize invasion. Spheroids were imaged at 0 and 24 hours after invasion using a Zeiss Axio Examiner Z1 microscope with $20 \times$ water immersion objective (1.0 NA DIC [UV] VIS-IR). The SHG signal was obtained using a band pass 380-430 $\mathrm{nm}^{3}$. To image the cells stained with Red CellTracker, we used a band pass of 570-610 nm cube with a long pass of $555 \mathrm{~nm}$. Images were taken with a Coherent Chameleon Verdi laser at $790 \mathrm{~nm}$ wavelength. Z-stack images were taken with a $1-\mu \mathrm{m}$ interval. Mouse tissue sections were imaged as above but at $820 \mathrm{~nm}$ wavelength. For quantitative comparisons, image acquisition settings were kept constant. Collagen SHG images were analyzed using FIJI (ImageJ) by calculating the mean pixel intensity across the entire image.

\section{Human lung tumor tissue}

Human lung adenocarcinoma tissue is a mixed cohort obtained from Emory University and WellStar Health System tumor bank tissue and Emory clinical FFPE tumor specimens. The former cohort was sequenced using a custom-designed standard NGS workflow for analyzing mutations in 17 genes, including $K R A S$, and the entire coding region and $30 \mathrm{bp}$ flanking each exon of STK11 (LKB1). Emory clinical FFPE specimens were analyzed using NGS libraries prepared with the Tru-Sight Tumor-26 kit (exons 1, 4, 6, 8 of STK11) and sequenced on the Illumina MiSeq (26). pYFAK IHC was scored on a scale from 0-3, and diffuse staining was defined as a score that represented greater than $50 \%$ of tumor cells. Focal upregulation was defined as a higher score in less than $50 \%$ of the tumor cells as compared with the score of the surrounding tissue.

\section{Statistics}

Prism software was used to generate and analyze the Pearson correlations (Figure 3, B and D) and to generate $P$ values using the one-way ANOVA with Tukey's multiple comparison test, the 2-tailed student's $t$ test, and the Fisher's exact tests. A $P$ value of less than 0.05 was considered significant. Statistical analysis for analyzing days from enrollment to onset of clinical symptoms in the long-term treatment trial was conducted using SAS version 9.3 and SAS macros developed by the Biostatistics 
and Bioinformatics Shared Resource at Winship Cancer Institute (27). The association of treatment with time to onset of clinical symptoms was assessed using the Cox Proportional hazards model. As symptoms related to treatment are expected to develop early, a Wilcoxon test was done to compare the Kaplan-Meier survival curves. Due to the relatively small sample size, a $P$ value of less than 0.1 was considered significant.

\section{Study Approval}

The Emory Institutional Care and Use Committee (IACUC) approved all animal studies and procedures. Studies performed using human lung adenocarcinoma tissue was performed with approval from the Emory University IRB and in concordance with regulatory guidelines regarding clinical assay validation. Informed consent was obtained from all patients, and the investigator performing the IHC was blinded to the identity of the samples.

\section{Author contributions}

WZ, AIM, and MGR designed the study, interpreted all data, and wrote the manuscript. MGR, J. Konen, J. Koo, JS, WGW, CH, and WDM performed the experiments. MR, ZC, and J. Kowlaski performed the statistical analyses. BSR and GLS scored the human and mouse pathology specimens. MB assisted with obtaining human tumor specimens. ALK provided the KP lung adenocarcinoma specimens. GHS, CEH, and MRR led the information technology, molecular pathology, and sequencing workflow for the human lung adenocarcinoma tissues. SSR, HF, and FRK participated in study design and edited the manuscript.

\section{Acknowledgments}

Glaxo Smith Kline provided the GSK6098 FAK inhibitor. This work was supported by grants P01CA116676 (FRK), R01CA142858 (AIM), R01CA140571 (WZ), R00CA178091 (ALK), R01CA194027 (WZ, AIM, MGR) and R01CA201340 (AIM, MGR) from the US National Institutes of Health National Cancer Institute. Research reported in this publication was supported in part by the Biostatistics and Bioinformatics Shared Resource, the Emory Integrated Cellular Imaging Core, and the Cancer Tissue and Pathology Core of the Winship Cancer Institute of Emory University and NIH/NCI under award number P30CA138292 (WJC). The content is solely the responsibility of the authors and does not necessarily represent the official views of the NIH. Lentiviral-Cre was obtained from the Emory Viral Vector Core, supported by the Emory Neuroscience NINDS Core Facilities grant P30NS055077.

Address correspondence to: Melissa Gilbert-Ross, Adam I. Marcus, or Wei Zhou, 1365C Clifton Road, C4090, Atlanta, Georgia 30322, USA. Phone: 404.712.8370; E-mail: mmgilbe@emory.edu (MGR). Phone: 404.778.4597; E-mail: aimarcu@emory.edu (AIM). Phone: 404.778.2134; E-mail: wzhou2@emory.edu (WZ). Or to: Fadlo R. Khuri, PO Box 11-0236, Riad E1 Solh 1107 2020, Beirut, Lebanon. Phone: 961.1.347127; E-mail: frkhuri@aub.edu.lb.

FRK's present address is: The American University of Beirut, Beirut, Lebanon.

1. Sanchez-Cespedes M, et al. Inactivation of LKB1/STK11 is a common event in adenocarcinomas of the lung. Cancer Res. 2002;62(13):3659-3662.

2. Carretero J, Medina PP, Pio R, Montuenga LM, Sanchez-Cespedes M. Novel and natural knockout lung cancer cell lines for the LKB1/STK11 tumor suppressor gene. Oncogene. 2004;23(22):4037-4040.

3. Ding L, et al. Somatic mutations affect key pathways in lung adenocarcinoma. Nature. 2008;455(7216):1069-1075.

4. Imielinski M, et al. Mapping the hallmarks of lung adenocarcinoma with massively parallel sequencing. Cell. 2012;150(6):1107-1120

5. Cancer Genome Atlas Research Network. Comprehensive molecular profiling of lung adenocarcinoma. Nature. 2014;511(7511):543-550.

6. Ji H, et al. LKB1 modulates lung cancer differentiation and metastasis. Nature. 2007;448(7155):807-810.

7. Chen $\mathrm{Z}$, et al. A murine lung cancer co-clinical trial identifies genetic modifiers of therapeutic response. Nature. 2012;483(7391):613-617.

8. Liu Y, et al. Metabolic and functional genomic studies identify deoxythymidylate kinase as a target in LKB1-mutant lung cancer. Cancer Discov. 2013;3(8):870-879.

9. Shackelford DB, et al. LKB1 inactivation dictates therapeutic response of non-small cell lung cancer to the metabolism drug phenformin. Cancer Cell. 2013;23(2):143-158. 
10. Konstantinidou G, et al. RHOA-FAK is a required signaling axis for the maintenance of KRAS-driven lung adenocarcinomas. Cancer Discov. 2013;3(4):444-457.

11. Carretero J, et al. Integrative genomic and proteomic analyses identify targets for Lkb1-deficient metastatic lung tumors. Cancer Cell. 2010;17(6):547-559.

12. Schabath MB, et al. Differential association of STK11 and TP53 with KRAS mutation-associated gene expression, proliferation and immune surveillance in lung adenocarcinoma. Oncogene. 2016;35(24):3209-3216.

13. Skoulidis F, et al. Co-occurring genomic alterations define major subsets of KRAS-mutant lung adenocarcinoma with distinct biology, immune profiles, and therapeutic vulnerabilities. Cancer Discov. 2015;5(8):860-877.

14. Gao Y, et al. LKB1 inhibits lung cancer progression through lysyl oxidase and extracellular matrix remodeling. Proc Natl Acad Sci USA. 2010;107(44):18892-18897.

15. Koyama S, et al. STK11/LKB1 Deficiency Promotes Neutrophil Recruitment and Proinflammatory Cytokine Production to Suppress T-cell Activity in the Lung Tumor Microenvironment. Cancer Res. 2016;76(5):999-1008.

16. Kline ER, Shupe J, Gilbert-Ross M, Zhou W, Marcus AI. LKB1 represses focal adhesion kinase (FAK) signaling via a FAK LKB1 complex to regulate FAK site maturation and directional persistence. J Biol Chem. 2013;288(24):17663-17674.

17. Goodwin JM, Svensson RU, Lou HJ, Winslow MM, Turk BE, Shaw RJ. An AMPK-independent signaling pathway downstream of the LKB1 tumor suppressor controls Snaill and metastatic potential. Mol Cell. 2014;55(3):436-450.

18. Konen J, et al. LKB1 kinase-dependent and -independent defects disrupt polarity and adhesion signaling to drive collagen remodeling during invasion. Mol Biol Cell. 2016;27(7):1069-1084.

19. Sulzmaier FJ, Jean C, Schlaepfer DD. FAK in cancer: mechanistic findings and clinical applications. Nat Rev Cancer. 2014;14(9):598-610.

20. Provenzano PP, Eliceiri KW, Campbell JM, Inman DR, White JG, Keely PJ. Collagen reorganization at the tumor-stromal interface facilitates local invasion. BMC Med. 2006;4(1):38.

21. Cheung KJ, Gabrielson E, Werb Z, Ewald AJ. Collective invasion in breast cancer requires a conserved basal epithelial program. Cell. 2013;155(7):1639-1651.

22. Fisher KE, et al. Clinical Validation and Implementation of a Targeted Next-Generation Sequencing Assay to Detect Somatic Variants in Non-Small Cell Lung, Melanoma, and Gastrointestinal Malignancies. J Mol Diagn. 2016;18(2):299-315.

23. Jackson EL, et al. Analysis of lung tumor initiation and progression using conditional expression of oncogenic K-ras. Genes Dev. 2001;15(24):3243-3248.

24. Bardeesy N, et al. Loss of the Lkb1 tumour suppressor provokes intestinal polyposis but resistance to transformation. Nature. 2002;419(6903):162-167.

25. Safran M, Kim WY, Kung AL, Horner JW, DePinho RA, Kaelin WG. Mouse reporter strain for noninvasive bioluminescent imaging of cells that have undergone Cre-mediated recombination. Mol Imaging. 2003;2(4):297-302.

26. Platt RJ, et al. CRISPR-Cas9 knockin mice for genome editing and cancer modeling. Cell. 2014;159(2):440-455.

27. Nickleach D, Liu Y, Shrewsberry A, Ogan K, Kim S, Wang Z. SAS ${ }^{\circ}$ Macros to Conduct Common Biostatistical Analyses and Generate Reports. Paper presnted at: SESUG 2013: The Proceeding of the Southeast SAS User Group; Ocotber 20-23, 2013; St. Pete Beach, FL. http://analytics.ncsu.edu/sesug/2013/PO-05.pdf. Accessed February 2, 2017. 